PROCEEDINGS OF THE

AMERICAN MATHEMATICAL SOCIETY

Volume 137, Number 8, August 2009, Pages 2591-2600

S 0002-9939(09)09854-2

Article electronically published on March 3, 2009

\title{
CYCLIC SHIFTS OF THE VAN DER CORPUT SET
}

\author{
DMITRIY BILYK
}

(Communicated by Michael T. Lacey)

\begin{abstract}
In 1980, K. Roth showed that the expected value of the $L^{2}$ discrepancy of the cyclic shifts of the $N$-point van der Corput set is bounded by a constant multiple of $\sqrt{\log N}$, thus guaranteeing the existence of a shift with asymptotically minimal $L^{2}$ discrepancy. In the present paper, we construct a specific example of such a shift.
\end{abstract}

\section{INTRODUCTION}

Let $\mathcal{A}_{N} \subset[0,1]^{2}$ be a finite point set of cardinality $N$. The extent of equidistribution of $\mathcal{A}_{N}$ can be measured by the discrepancy function:

$$
D_{\mathcal{A}_{N}}\left(x_{1}, x_{2}\right):=\sharp\left(\mathcal{A}_{N} \cap\left[0, x_{1}\right) \times\left[0, x_{2}\right)\right)-N x_{1} \cdot x_{2},
$$

i.e. the difference between the actual and expected number of points of $\mathcal{A}_{N}$ in the rectangle $\left[0, x_{1}\right) \times\left[0, x_{2}\right)$. The main principle of the theory of irregularities of distribution states that the size of this function must increase with $N$. The fundamental results in the subject are:

K. Roth's Theorem $\left([11,1954)\right.$. For any set $\mathcal{A}_{N} \subset[0,1]^{2}$, we have

$$
\left\|D_{\mathcal{A}_{N}}\right\|_{2} \gtrsim(\log N)^{1 / 2} \text {, }
$$

where " $\gtrsim$ " stands for "greater than a constant multiple of".

W. Schmidt's Theorem $\left([14,1972)\right.$. For any set $\mathcal{A}_{N} \subset[0,1]^{2}$, we have

$$
\left\|D_{\mathcal{A}_{N}}\right\|_{\infty} \gtrsim \log N \text {. }
$$

Both theorems are known to be sharp in the order of magnitude (e.g., 6], 7], [12, 2]). One of the most famous examples, yielding sharpness of (1.2), is the van der Corput "digit-reversing" set, 6]. For $N=2^{n}$ points, it can be defined as

$$
\mathcal{V}_{n}=\left\{\left(0 . a_{1} a_{2} \ldots a_{n} 1,0 . a_{n} a_{n-1} \ldots a_{2} a_{1} 1\right): a_{i}=0,1\right\}
$$

where the coordinates are given in terms of the binary expansion. Unfortunately, most "classical" sets with minimal $L^{\infty}$ norm of the discrepancy fail to meet the sharp bounds in the $L^{2}$ norm. In fact, Halton and Zaremba $[8]$ proved that

$$
\left\|D_{\mathcal{V}_{n}}\right\|_{2}^{2}=\frac{n^{2}}{2^{6}}+O(n) \approx(\log N)^{2} .
$$

Received by the editors October 22, 2008

2000 Mathematics Subject Classification. Primary 11K38; Secondary 42B05.

Key words and phrases. Discrepancy theory, Fourier analysis.

The author is grateful to the Fields Institute and the Institute for Advanced Study for hospitality and to the National Science Foundation for support (grants DMS-0801036 and DMS-0635607).

(C)2009 American Mathematical Society 2591

Reverts to public domain 28 years from publication 
There are three standard remedies in the theory for this shortcoming. To achieve the smallest possible order of the $L^{2}$ discrepancy, one can alter the sets in the following ways:

1. Davenport's Reflection Principle. Informally, if $P$ has low $L^{\infty}$ discrepancy, then the set $\widetilde{P}=P \cup\{(1-x, y):(x, y) \in P\}$ has low $L^{2}$ discrepancy. This was demonstrated by Davenport [7] in the case of the irrational lattice, and by Chen and Skriganov ([5], see also [10]) in the case of the van der Corput set.

2. Digit scrambling. This procedure, initially introduced in [3], has been extensively studied; a comprehensive discussion can be found in 9 .

3. Cyclic shifts. This transformation is the subject of this paper. It has been proved by Roth, [13] (see also [12, where the translation idea was originally used) that for the cyclic shifts of the van der Corput set

$$
\mathcal{V}_{n}^{\alpha}=\left\{((x+\alpha) \bmod 1, y):(x, y) \in \mathcal{V}_{n}\right\},
$$

the expected value of the $L^{2}$ discrepancy over $\alpha$ satisfies

$$
\int_{0}^{1}\left\|D_{\mathcal{V}_{n}^{\alpha}}\right\|_{2} d \alpha \lesssim n^{1 / 2}=(\log N)^{1 / 2}
$$

This implies that there exists a particular cyclic shift of the van der Corput distribution with minimal $L^{2}$ norm of the discrepancy function. However, this was purely an existence proof, and no deterministic examples of such shifts have been constructed. In the present paper, we "de-randomize" this result and provide an explicit value of $\alpha$, which asymptotically minimizes $\left\|D_{\mathcal{V}_{n}^{\alpha}}\right\|_{2}$. We prove the following theorem.

Theorem 1.1. For $\alpha_{0}=1-\frac{k}{2^{n}}$, where $k \in \mathbb{N}$, in the binary form, is given by

$$
k:=(\underbrace{000 \ldots 00}_{n_{0} \text { digits }} \underbrace{00001111 \ldots 00001111}_{n_{2} \text { digits }} \underbrace{000111 \ldots 000111}_{n_{1} \text { digits }})_{2}+1,
$$

with $n_{0}+n_{1}+n_{2}=n, \frac{n_{1}}{n_{2}}=\frac{54}{17}$, and $n_{0}<568$, the cyclically shifted van der Corput set $\mathcal{V}_{n}^{\alpha_{0}}$ satisfies

$$
\left\|D_{\mathcal{V}_{n}^{\alpha}}\right\|_{2} \lesssim n^{1 / 2}=(\log N)^{1 / 2}
$$

Remark. The " +1 " at the end of (1.7) is just a minor nuisance, which simplifies some calculations, and is not important. In fact, one can easily see that a cyclic shift by the amount $\alpha=1 / N=2^{-n}$ changes the discrepancy by at most 1 at each point.

We would like to point out that most constructions of sets with minimal order of $L^{p}$ discrepancy (which are important in applications to numerical integration) are probabilistic; explicit constructions are rare. In fact, the first deterministic examples of such sets in dimensions $d \geq 3$ have only been obtained quite recently by Chen and Skriganov (4, [15).

The outline of the paper is the following: In $\S 2$ we deal with the quantities $\int_{[0,1]^{2}} D_{\mathcal{V}_{n}}(x) d x$ and $\int_{[0,1]^{2}} D_{\mathcal{V}_{n}^{\alpha}}(x) d x$ (which can be viewed as the "zero-order" term of the expansion in any reasonable orthonormal basis) and minimize the latter. In $\S 3$, we examine the Fourier coefficients $\widehat{D_{\mathcal{V}_{n}}}\left(n_{1}, n_{2}\right)$ when $\left(n_{1}, n_{2}\right) \neq(0,0)$ and show that they do not change too much under cyclic shifts. 
We will refer to the two parts of the discrepancy function as "linear" and "counting":

$$
\begin{aligned}
L_{N}\left(x_{1}, x_{2}\right) & =N x_{1} \cdot x_{2}, \\
C_{\mathcal{A}_{N}}\left(x_{1}, x_{2}\right) & =\sum_{p \in \mathcal{A}_{N}} \mathbf{1}_{\left[p_{1}, 1\right) \times\left[p_{2}, 1\right)}\left(x_{1}, x_{2}\right) .
\end{aligned}
$$

In proving upper bounds for the discrepancy function, one of course needs to capture a large cancelation between these two.

\section{The INTEGRAL OF THE DISCREPANCY FUNCTION}

Recall that in our definition of the van der Corput set, $\mathcal{V}_{n}=$ $\left\{\left(0 . a_{1} \ldots a_{n} 1,0 . a_{n} \ldots a_{2} a_{1} 1\right)\right\}$, both coordinates have 1's in the $(n+1)^{s t}$ binary place. This is just a technical modification, which ensures that, for any $\alpha=j / 2^{n}$, $j \in \mathbb{Z}$, the average value of both coordinates in $\mathcal{V}_{n}^{\alpha}$ is one-half:

$$
\frac{1}{2^{n}} \sum_{\left(p_{1}, p_{2}\right) \in \mathcal{V}_{n}^{\alpha}} p_{1}=\frac{1}{2^{n}} \sum_{\left(p_{1}, p_{2}\right) \in \mathcal{V}_{n}^{\alpha}} p_{2}=\frac{1}{2}
$$

This makes many formulas look 'cleaner' and is not essential to the computations.

It has been noticed (see [8], 1]), that the quantity $\int_{[0,1]^{2}} D_{\mathcal{V}_{n}}(x) d x$ is the main reason why $\left\|D_{\mathcal{V}_{n}}\right\|_{2}$ is large. Indeed, if one compares (1.4) and (2.3) below, it is easy to see that

$$
\left\|D_{\mathcal{V}_{n}}-\int D_{\mathcal{V}_{n}}\right\|_{2} \lesssim(\log N)^{1 / 2}
$$

We include the proof of the lemma below for the sake of completeness.

Lemma 2.1. For the van der Corput set $\mathcal{V}_{n}$,

$$
\int_{[0,1)^{2}} D_{\mathcal{V}_{n}}(x) d x=\frac{n}{8} .
$$

Proof. The linear part of the discrepancy function clearly gives us

$$
\int_{[0,1)^{2}} L_{N} d x=2^{n-2}
$$

Let $X_{1}, \ldots, X_{n}$ be independent random variables taking values $\{0,1\}$ with probability $\frac{1}{2}$. A straightforward computation yields

$$
\begin{aligned}
& \int_{[0,1)^{2}} C_{\mathcal{V}_{n}}\left(x_{1}, x_{2}\right) d x_{1} d x_{2}=\sum_{\left(p_{1}, p_{2}\right) \in \mathcal{V}_{n}}\left(1-p_{1}\right)\left(1-p_{2}\right) \\
& =2^{n} \mathbb{E}\left[1-\sum_{j=1}^{n} X_{j} 2^{-j}-2^{-n-1}\right]\left[1-\sum_{k=1}^{n} X_{k} 2^{-n+k-1}-2^{-n-1}\right] \\
& =2^{n-2}+\frac{n}{8} .
\end{aligned}
$$

Combining (2.4) and (2.5) proves the lemma.

In what follows we prove that the average of $\int_{[0,1]^{2}} D_{\mathcal{V}_{n}^{\alpha}} d x$ over $\alpha$ is zero. Besides, we construct a specific value of $\alpha_{0}$, for which

$$
\int_{[0,1]^{2}} D_{\mathcal{V}_{n}^{\alpha_{0}}} d x \approx 1
$$


Theorem 2.2. Assume that $\alpha \in[0,1)$ is an n-digit binary number. Then

$$
\mathbb{E}_{\alpha} \int_{[0,1]^{2}} D_{\mathcal{V}_{n}^{\alpha}} d x=0
$$

Proof. We denote $1-\alpha=\frac{k}{2^{n}}\left(k=1, \ldots, 2^{n}\right)$ and start with the following computation:

$$
\begin{aligned}
\int_{[0,1]^{2}} C_{\mathcal{V}_{n}^{\alpha}} & =\sum_{p \in \mathcal{V}_{n}^{\alpha}}\left(1-p_{1}\right)\left(1-p_{2}\right) \\
& =\sum_{p \in \mathcal{V}_{n}: p_{1}<1-\alpha}\left(1-p_{1}-\alpha\right) \cdot\left(1-p_{2}\right)+\sum_{p \in \mathcal{V}_{n}: p_{1}>1-\alpha}\left(2-p_{1}-\alpha\right) \cdot\left(1-p_{2}\right) \\
& =\int_{[0,1]^{2}} C_{\mathcal{V}_{n}} d x+(1-\alpha) \sum_{p \in \mathcal{V}_{n}}\left(1-p_{2}\right)-\sum_{p \in \mathcal{V}_{n}: p_{1}<1-\alpha}\left(1-p_{2}\right) \\
& =\int_{[0,1]^{2}} C_{\mathcal{V}_{n}} d x-\frac{k}{2}+\sum_{p \in \mathcal{V}_{n}: p_{1}<k / 2^{n}} p_{2} .
\end{aligned}
$$

Next, we examine the behavior of the last sum above. Using the structure of the van der Corput set, we can write

$$
\sum_{p \in \mathcal{V}_{n}: p_{1}<k / 2^{n}} p_{2}=\sum_{l=1}^{n} 2^{-l} f_{l}(k)+k 2^{-n-1},
$$

where $k 2^{-n-1}$ comes from the final 1's in the expansion of $p_{2}$ and

$f_{l}(k)=\#\left\{0 \leq j \leq k-1\right.$ such that the $l^{\text {th }}$ (from the end) binary digit of $j$ is 1$\}$.

It can be seen that

$$
\begin{array}{cl}
f_{l}(k)=2^{l-1} m & \text { if } k-1=2^{l} m, 2^{l} m+1, \ldots, 2^{l} m+2^{l-1}-1, \text { and } \\
f_{l}(k)=2^{l-1} m+j & \text { if } k-1=2^{l} m+2^{l-1}+j-1, \text { where } 1 \leq j \leq 2^{l-1}
\end{array}
$$

Thus, if we set $f_{l}(k)=2^{l-1} m_{l}(k)+j_{l}(k)$, where $0 \leq m_{l}(k)<2^{n-l}$ and $1 \leq j_{l}(k) \leq$ $2^{l-1}$, we have $\mathbb{E}_{k} m_{l}(k)=\frac{1}{2} \cdot\left(2^{n-l}-1\right)$ and

$$
\mathbb{E}_{k} j_{l}(k)=\frac{1}{2} \cdot \frac{1}{2}\left(2^{l-1}+1\right),
$$

where the extra one-half above comes from the fact that $j_{l}(k)=0$ half of the time. Thus

$$
\mathbb{E}_{k} f_{l}(k)=2^{n-2}-2^{l-2}+2^{l-3}+\frac{1}{4} .
$$

Plugging this into (2.8), we obtain

$$
\begin{aligned}
\mathbb{E}_{k} \sum_{p \in \mathcal{V}_{n}: p_{1}<k / 2^{n}} p_{2} & =\sum_{l=1}^{n} 2^{-l}\left(2^{n-2}-2^{l-3}+\frac{1}{4}\right)+\mathbb{E}_{k} k \cdot 2^{-n-1} \\
& =2^{n-2}-\frac{n}{8}+\frac{1}{4} .
\end{aligned}
$$


Finally, equation (2.7), together with (2.13) as well as (2.3), yields

$$
\begin{aligned}
\mathbb{E} \int_{[0,1]^{2}} D_{\mathcal{V}_{n}^{\alpha}} & =\int_{[0,1]^{2}} D_{\mathcal{V}_{n}} d x-\mathbb{E}_{k} \frac{k}{2}+\mathbb{E}_{k} \sum_{p \in \mathcal{V}_{n}: p_{1}<k / 2^{n}} p_{2} \\
& =\frac{n}{8}-\left(2^{n-2}+\frac{1}{4}\right)+\left(2^{n-2}-\frac{n}{8}+\frac{1}{4}\right)=0 .
\end{aligned}
$$

To facilitate the construction of an example, we further look at the functions $f_{l}(k)=2^{l-1} m_{l}(k)+j_{l}(k)$ defined above, (2.9)-(2.11). Assume that $k-1$ is written in the binary representation:

$$
k-1=\sum_{j=1}^{n} k_{j} \cdot 2^{j-1}=\left(k_{n} k_{n-1} \ldots k_{2} k_{1}\right)_{2} .
$$

By construction, $m_{l}(k)=\left(k_{n} k_{n-1} \ldots k_{l+1}\right)_{2}$; furthermore, when $k_{l}=0$, we have $j_{l}(k)=0$, and if $k_{l}=1, j_{l}(k)=\left(k_{l-1} \ldots k_{1}\right)_{2}+1$. Thus, $f_{l}(k)$ can be written in closed form in terms of the digits of $k-1$ as follows:

$$
f_{l}(k)=\sum_{j=l+1}^{n} k_{j} 2^{j-2}+k_{l} \cdot \sum_{j=1}^{l-1} k_{j} 2^{j-1}+k_{l} .
$$

Indeed, if $k_{l}=0$, the last two terms will disappear; otherwise, they'll equal exactly $j_{l}(k)$.

Plugging this into (2.8), we obtain

$$
\sum_{p \in \mathcal{V}_{n}: p_{1}<k / 2^{n}} p_{2}=\sum_{l=1}^{n-1} \sum_{j=l+1}^{n} k_{j} \cdot 2^{j-l-2}+\sum_{l=1}^{n} k_{l} \cdot 2^{-l}+\sum_{l=2}^{n} \sum_{j=1}^{l-1} k_{j} \cdot k_{l} \cdot 2^{j-l-1} .
$$

Obviously, the second term above is bounded by one. Next we shall look at the first term in (2.16). At this point we assume that

$$
\sum_{j=1}^{n} k_{j}=\frac{n}{2}+O(1)
$$

i.e. approximately half of the binary digits of $k-1$ are ones and half are zeros. We have

$$
\begin{aligned}
\sum_{l=1}^{n-1} \sum_{j=l+1}^{n} k_{j} \cdot 2^{j-l-2} & =\frac{1}{2} \sum_{j=2}^{n} k_{j} \cdot 2^{j-1} \sum_{l=1}^{j-1} 2^{-l}=\frac{1}{2} \sum_{j=2}^{n} k_{j} \cdot 2^{j-1} \cdot\left(1-2^{-(j-1)}\right) \\
& =\frac{1}{2} \sum_{j=2}^{n} k_{j} \cdot 2^{j-1}-\frac{1}{2} \sum_{j=2}^{n} k_{j}=\frac{1}{2} k-\frac{n}{4}+O(1) .
\end{aligned}
$$

As to the last term of (2.16), we have the following lemma:

Lemma 2.3. For every $n \in \mathbb{N}$, there exists $k: 1 \leq k \leq 2^{n}$ with $\sum_{j=1}^{n} k_{j}=$ $n / 2+O(1)$, where $k-1=\left(k_{n} k_{n-1} \ldots k_{2} k_{1}\right)_{2}$, so that

$$
\sum_{l=2}^{n} \sum_{j=1}^{l-1} k_{j} \cdot k_{l} \cdot 2^{j-l-1}=\frac{n}{8}+O(1) \text {. }
$$


Assuming this statement for the moment and putting together (2.16), (2.18), and (2.19) for $k$ defined by Lemma 2.3 above, we obtain

$$
\sum_{p \in \mathcal{V}_{n}: p_{1}<k / 2^{n}} p_{2}=\left(\frac{1}{2} k-\frac{n}{4}\right)+\frac{n}{8}+O(1)=\frac{1}{2} k-\frac{n}{8}+O(1) .
$$

Together with (2.7), (2.5), this yields:

$$
\int_{[0,1]^{2}} C_{\mathcal{V}_{n}^{\alpha}} d x=\left(2^{n-2}+\frac{n}{8}\right)-\frac{1}{2} k+\left(\frac{1}{2} k-\frac{n}{8}\right)+O(1)=2^{n-2}+O(1) .
$$

Finally, (2.21) and (2.4) give

$$
\int_{[0,1]^{2}} D_{\mathcal{V}_{n}^{\alpha}}(x) d x=O(1)
$$

Thus, it remains to prove Lemma 2.3. We shall denote

$$
S(n, k-1):=\sum_{l=2}^{n} \sum_{j=1}^{l-1} k_{j} \cdot k_{l} \cdot 2^{j-l-1}
$$

and will look at some basic examples first. Let $k^{\prime}$ be of the form

$$
k^{\prime}:=(000111 \ldots 000111)_{2},
$$

where the sequence 000111 is repeated $n^{\prime}$ times, $n=6 n^{\prime}$. We then have the following calculation:

$S\left(6 n^{\prime}, k^{\prime}\right)=\frac{1}{2} \sum_{l^{\prime}=1}^{n^{\prime}-1}\left(2^{-\left(6 l^{\prime}+1\right)}+2^{-\left(6 l^{\prime}+2\right)}+2^{-\left(6 l^{\prime}+3\right)}\right)\left(\sum_{j^{\prime}=0}^{l^{\prime}-1}\left[2^{6 j^{\prime}+1}+2^{6 j^{\prime}+2}+2^{6 j^{\prime}+3}\right]\right)$

$$
\begin{aligned}
& +\frac{1}{2} \sum_{l^{\prime}=0}^{n^{\prime}-1}\left(\frac{1}{2}+\left(\frac{1}{2}+\frac{1}{4}\right)\right) \\
= & \frac{1}{2} \sum_{l^{\prime}=1}^{n^{\prime}-1} 2^{-6 l^{\prime}}\left(2^{6 l^{\prime}}-1\right) \frac{1}{2^{6}-1}\left(2^{-1}+2^{-2}+2^{-3}\right)\left(2^{1}+2^{2}+2^{3}\right)+\frac{1}{2} \cdot \frac{5}{4} n^{\prime} \\
= & \left(\frac{7}{72}+\frac{45}{72}\right) \cdot \frac{n}{6}+O(1) \\
= & \frac{13}{108} n+O(1),
\end{aligned}
$$

where the term in (2.25) describes the interactions of digits in different triples and (2.26) arises from interactions within the triples. (Notice that the obtained fraction $\frac{13}{108} \approx 0.12037 \ldots$ is quite close to the desired $\frac{1}{8}=0.125$.) 
Next we set $k^{\prime \prime}=(00001111 \ldots . .00001111)_{2}$, where the string 00001111 is repeated $n^{\prime \prime}$ times. An absolutely analogous computation yields:

$$
\begin{aligned}
& S\left(8 n^{\prime \prime}, k^{\prime \prime}\right) \\
& =\frac{1}{2} \sum_{l^{\prime \prime}=1}^{n^{\prime \prime}-1} 2^{-8 l^{\prime \prime}}\left(2^{8 l^{\prime \prime}}-1\right) \frac{1}{2^{8}-1}\left(2^{-1}+2^{-2}+2^{-3}+2^{-4}\right)\left(2^{1}+2^{2}+2^{3}+2^{4}\right) \\
& \quad+\frac{1}{2} \sum_{l^{\prime}=0}^{n^{\prime}-1}\left(\frac{1}{2}+\left(\frac{1}{2}+\frac{1}{4}\right)+\left(\frac{1}{2}+\frac{1}{4}+\frac{1}{8}\right)\right) \\
& =\frac{19}{136} n+O(1) .
\end{aligned}
$$

We are now ready to define the number $k$ which satisfies (2.19) . Set

$$
k-1:=(\underbrace{00001111 \ldots 00001111}_{n_{2} \text { digits }} \underbrace{000111 \ldots 000111}_{n_{1} \text { digits }})_{2} .
$$

Then we have

$$
S\left(n_{1}+n_{2}, k-1\right)=S\left(n_{1}, k^{\prime}\right)+S\left(n_{2}, k^{\prime \prime}\right)+I\left(n_{1}, n_{2}\right),
$$

where $I\left(n_{1}, n_{2}\right)$ describes the interaction between the two parts of $k$. We can estimate:

$$
I\left(n_{1}, n_{2}\right)=\frac{1}{2}\left(\sum_{l=n_{1}+1}^{n} k_{l} 2^{-l}\right)\left(\sum_{j=1}^{n_{1}} k_{j} 2^{j}\right) \leq \frac{1}{2}\left(2^{-n_{1}-1} \cdot 2\right)\left(2^{n_{1}+1}-1\right) \leq 1 .
$$

We now choose $n_{1}$ and $n_{2}$ so that $\frac{n_{1}}{n_{2}}=\frac{54}{17}$, i.e. $n_{1}=\frac{54}{71} n, n_{2}=\frac{17}{71} n$. We then obtain

$$
\begin{aligned}
S(n, k-1) & =\frac{13}{108} n_{1}+\frac{19}{136} n_{2}+O(1) \\
& =\left(\frac{13 \cdot 54}{108 \cdot 71}+\frac{19 \cdot 17}{136 \cdot 71}\right) n+O(1) \\
& =\frac{n}{8}+O(1),
\end{aligned}
$$

which finishes the proof of Lemma 2.3. Thus, if we set $\alpha_{0}=1-\frac{k}{2^{n}}$, where $k$ is as defined in (2.29), then the cyclic shift of the van der Corput set by $\alpha_{0}$ satisfies

$$
\int_{[0,1]^{2}} D_{\mathcal{V}_{n}^{\alpha_{0}}}(x) d x=O(1) .
$$

Remark. Of course, the above construction only works when $n$ is a multiple of $71 \cdot 2 \cdot 4=568$. However, it can be easily adjusted for other values of $n$ just by setting the "remainder" digits equal to zero.

\section{The Fourier COEFFICIENTS OF THE DISCREPANCY FUnCTION}

Having eliminated the main problem, we shall now proceed to show that the remaining part of $D_{\mathcal{V}_{n}}$ behaves well under cyclic shifts. We shall use the exponential Fourier basis (rather than the more standard in this theory Haar basis) since it is better adapted to cyclic shifts. 
Obviously, for any $\alpha$, we have

$$
\begin{aligned}
\sum_{p \in \mathcal{V}_{n}^{\alpha}} e^{-2 \pi i m p_{1}} & =\sum_{p \in \mathcal{V}_{n}} e^{-2 \pi i m p_{1}}=\sum_{j=0}^{2^{n}-1} e^{-2 \pi i \frac{m}{2^{n}} j} \cdot e^{-\pi i \frac{m}{2^{n}}} \\
& = \begin{cases}0, & \text { if } m \neq \equiv \quad \bmod 2^{n} \\
m, & \text { if } m=2^{n} m^{\prime}, m^{\prime} \text { even, } \\
-m, & \text { if } m=2^{n} m^{\prime}, m^{\prime} \text { odd. }\end{cases}
\end{aligned}
$$

Fourier coefficients in the case $n_{1}, n_{2} \neq 0$. We first note that, for $n_{1}, n_{2} \neq 0$, the Fourier coefficient of the linear part is:

$$
\widehat{L_{N}}\left(n_{1}, n_{2}\right)=-\frac{N}{4 \pi^{2} n_{1} n_{2}} .
$$

The counting part yields

$$
\widehat{C_{\mathcal{V}_{N}}}\left(n_{1}, n_{2}\right)=-\frac{1}{4 \pi^{2} n_{1} n_{2}} \sum_{p \in \mathcal{V}_{N}}\left(1-e^{-2 \pi i n_{1} p_{1}}\right)\left(1-e^{-2 \pi i n_{2} p_{2}}\right),
$$

and, thus,

$$
\widehat{D_{\mathcal{V}_{n}}}\left(n_{1}, n_{2}\right)=\frac{1}{4 \pi^{2} n_{1} n_{2}} \sum_{p \in \mathcal{V}_{N}}\left(e^{-2 \pi i n_{1} p_{1}}+e^{-2 \pi i n_{2} p_{2}}-e^{-2 \pi i\left(n_{1} p_{1}+n_{2} p_{2}\right)}\right) .
$$

We now consider the following cases:

- Both $n_{1}$ and $n_{2} \equiv 0 \bmod 2^{n}$. Then $\widehat{D_{\mathcal{V}_{n}}}\left(n_{1}, n_{2}\right)=C \frac{N}{4 \pi^{2} n_{1} n_{2}}$, where $C$ takes values -3 or 1 , depending on whether $n_{1} / 2^{n}$ and $n_{2} / 2^{n}$ are even or odd.

- $n_{1} \not \equiv 0 \bmod 2^{n}, n_{2} \equiv 0 \bmod 2^{n}$. In this case $\widehat{D_{\mathcal{V}_{n}}}\left(n_{1}, n_{2}\right)=\frac{N}{4 \pi^{2} n_{1} n_{2}}$. $e^{-\pi i n_{2} / 2^{n}}$

- $n_{2} \not \equiv 0 \bmod 2^{n}, n_{1} \equiv 0 \bmod 2^{n}$. In this case $\widehat{\overline{\mathcal{V}}_{n}}\left(n_{1}, n_{2}\right)=\frac{N}{4 \pi^{2} n_{1} n_{2}}$. $e^{-\pi i n_{1} / 2^{n}}$.

- $n_{1}, n_{2} \not \equiv 0 \bmod 2^{n}$. In this case we have that $\widehat{D_{\mathcal{V}_{n}}}\left(n_{1}, n_{2}\right)=$ $-\frac{1}{4 \pi^{2} n_{1} n_{2}} \sum_{p \in \mathcal{V}_{N}} e^{-2 \pi i\left(n_{1} p_{1}+n_{2} p_{2}\right)}$.

Changing $p_{1}$ to $\left(p_{1}+\alpha\right) \bmod 1$ in the above computations, with $\alpha=j / 2^{n}$, we notice that

$$
\left|\widehat{D_{\mathcal{V}_{n}^{\alpha}}}\left(n_{1}, n_{2}\right)\right|=\left|\widehat{D_{\mathcal{V}_{n}}}\left(n_{1}, n_{2}\right)\right| \text { when } n_{1}, n_{2} \neq 0 \text {. }
$$

Indeed, in the first three cases the coefficient does not change, while in the last it is multiplied by $e^{-2 \pi i n_{1} \alpha}$.

Fourier coefficients in the case $n_{2}=0, n_{1} \neq 0$. We first note that, in this case,

$$
\widehat{L_{N}}\left(n_{1}, 0\right)=-\frac{N}{4 \pi i n_{1}} \text { and } \widehat{C_{\mathcal{V}_{n}}}\left(n_{1}, 0\right)=-\frac{1}{2 \pi i n_{1}} \sum_{p \in \mathcal{V}_{n}}\left(1-e^{-2 \pi i n_{1} p_{1}}\right)\left(1-p_{2}\right) .
$$

Thus, taking into account (2.1), we have

$$
\widehat{D_{\mathcal{V}_{n}}}\left(n_{1}, 0\right)=\frac{1}{2 \pi i n_{1}} \sum_{p \in \mathcal{V}_{n}} e^{-2 \pi i n_{1} p_{1}} \cdot\left(1-p_{2}\right) .
$$


Once again, we obtain that

$$
\widehat{D_{\mathcal{V}_{n}^{\alpha}}}\left(n_{1}, 0\right)=\widehat{D_{\mathcal{V}_{n}}}\left(n_{1}, 0\right) \cdot e^{-2 \pi i n_{1} \alpha}, \quad \text { i.e. }\left|\widehat{D_{\mathcal{V}_{n}^{\alpha}}}\right|=\left|\widehat{D_{\mathcal{V}_{n}}}\right| \text { if } n_{1} \neq 0, n_{2}=0 \text {. }
$$

Fourier coefficients in the case $n_{1}=0, n_{2} \neq 0$. As above, we can compute

$$
\widehat{D_{\mathcal{V}_{n}}}\left(0, n_{2}\right)=\frac{1}{2 \pi i n_{2}} \sum_{p \in \mathcal{V}_{n}}\left(1-p_{1}\right) \cdot e^{-2 \pi i n_{2} p_{2}} .
$$

In the case $n_{2} \equiv 0 \bmod 2^{n}$, we obtain, using (2.1),

$$
\widehat{D_{\mathcal{V}_{n}}}\left(0, n_{2}\right)=\widehat{D_{\mathcal{V}_{n}^{\alpha}}}\left(0, n_{2}\right)=\frac{N}{4 \pi i n_{2}} \cdot e^{-\pi i n_{2} / 2^{n}} \text {. }
$$

The only somewhat non-trivial case is when $n_{1}=0, n_{2} \not \equiv 0 \bmod 2^{n}$. The Fourier coefficient in this case is

$$
\begin{aligned}
\widehat{D_{\mathcal{V}_{n}^{\alpha}}^{\alpha}}\left(0, n_{2}\right) & =\frac{1}{2 \pi i n_{2}} \sum_{p \in \mathcal{V}_{n}^{\alpha}}\left(1-p_{1}\right) \cdot e^{-2 \pi i n_{2} p_{2}} \\
& =\widehat{D_{\mathcal{V}_{n}}}\left(0, n_{2}\right)+\frac{1}{2 \pi i n_{2}} \sum_{p \in \mathcal{V}_{n}: p_{1}>k / 2^{n}} e^{-2 \pi i n_{2} p_{2}}, \quad \text { where } k / 2^{n}=1-\alpha .
\end{aligned}
$$

We shall examine the last sum above. Assume $n_{2}=2^{s} m$, where $0 \leq s<n$ and $m$ is odd. Let us look over the part of the sum, ranging over a dyadic interval of length $2^{-l}, 1 \leq l \leq n$. This means that the first $l$ digits of $p_{1}$ (and thus, the last $l$ digits of $p_{2}$ ) are fixed, and the last $n-l$ (the first $n-l$ of $p_{2}$ ) are allowed to change freely:

$$
\begin{aligned}
& \sum_{p \in \mathcal{V}_{n}: p_{1} \in\left[q 2^{-l},(q+1) 2^{-l}\right)} e^{-2 \pi i n_{2} p_{2}} \\
& =e^{-2 \pi i 2^{s} m\left(q_{n-l+1} 2^{-n+l-1}+\cdots+q_{n} 2^{-n}+2^{-n-1}\right)} \cdot \sum_{j=0}^{2^{n-l}-1} e^{-2 \pi i m 2^{-n+l+s} j} .
\end{aligned}
$$

It is easy to see that the last sum equals zero when $l+s<n$; otherwise, its absolute value is at most $2^{n-l}$. We now split the interval $\left\{p_{1}>k / 2^{n}\right\}$ into at most $n$ dyadic intervals of length $2^{-l}, 1 \leq l \leq n$. We obtain

$$
\left|\sum_{p \in \mathcal{V}_{n}: p_{1}>k / 2^{n}} e^{-2 \pi i n_{2} p_{2}}\right| \leq \sum_{l=n-s}^{n} 2^{n-l}=2^{s+1}-1 .
$$

That is, for $n_{2}=2^{s} m$, by (3.11) and (3.13), we have

$$
\left|\widehat{D_{\mathcal{V}_{n}^{\alpha}}^{\alpha}}\left(0, n_{2}\right)-\widehat{D_{\mathcal{V}_{n}}}\left(0, n_{2}\right)\right| \leq \frac{2^{s+1}}{2 \pi n_{2}}=\frac{1}{\pi m} .
$$

\section{Proof of Theorem 1.1}

For a function $f \in L^{2}\left([0,1]^{2}\right)$ and $S \subset \mathbb{Z}^{2}$, we shall denote by $f_{S}$ the orthogonal projection of $f$ onto the span of the Fourier terms with indices in $S$, i.e.

$$
f_{S}\left(x_{1}, x_{2}\right) \stackrel{\text { def }}{=} \sum_{\left(n_{1}, n_{2}\right) \in S} \widehat{f}\left(n_{1}, n_{2}\right) e^{2 \pi i\left(n_{1} x_{1}+n_{2} x_{2}\right)} .
$$


Due to (3.5), (3.8), and Parseval's identity, we have

$$
\left\|\left(D_{\mathcal{V}_{n}^{\alpha_{0}}}\right)_{\mathbb{Z}^{2} \backslash\left\{n_{1}=0\right\}}\right\|_{2}=\left\|\left(D_{\mathcal{V}_{n}}\right)_{\mathbb{Z}^{2} \backslash\left\{n_{1}=0\right\}}\right\|_{2} .
$$

Inequality (3.14) yields

$$
\left\|\left(D_{\mathcal{V}_{n}^{\alpha}}-D_{\mathcal{V}_{n}}\right)_{\left\{n_{1}=0, n_{2} \neq 0\right\}}\right\|_{2}^{2} \lesssim \sum_{s=0}^{n-1} \sum_{m \text { odd }} \frac{1}{m^{2}} \lesssim n=\log N .
$$

Thus, we see that $\left\|\left(D_{\mathcal{V}_{n}}\right)_{\mathbb{Z}^{2} \backslash(0,0)}\right\|_{2}$ indeed does not change much under cyclic shifts. The inequalities above and (2.2) yield:

$$
\left\|\left(D_{\mathcal{V}_{n}^{\alpha}}^{\alpha_{0}}\right)_{\mathbb{Z}^{2} \backslash(0,0)}\right\|_{2} \lesssim\left\|\left(D_{\mathcal{V}_{n}}\right)_{\mathbb{Z}^{2} \backslash(0,0)}\right\|_{2}+(\log N)^{1 / 2} \lesssim(\log N)^{1 / 2} .
$$

Together with the fact that $\int D_{\mathcal{V}_{n}^{\alpha_{0}}} \lesssim 1$, (2.33), this finishes the proof:

$$
\left\|D_{\mathcal{V}_{n}^{\alpha_{0}}}\right\|_{2} \lesssim(\log N)^{1 / 2} .
$$

\section{REFERENCES}

1. D. Bilyk, M. Lacey, I. Parissis, A. Vagharshakyan, Exponential squared integrability of the discrepancy function in two dimensions, to appear in Mathematika.

2. W.W.L. Chen, On irregularities of distribution, Mathematika 27 (1980), no. 2, 153-170 (1981). MR610701 (82i:10044)

3. W.W.L. Chen, On irregularities of distribution. II, Quart. J. Math. Oxford Ser. (2) 34 (1983), 257-279. MR:711520(85c:11065)

4. W.W.L Chen, M.M. Skriganov, Explicit constructions in the classical mean squares problem in irregularities of point distribution, J. Reine Angew. Math. 545 (2002), 67-95. MR1896098 (2003g:11083)

5. W.W.L Chen, M.M. Skriganov, Davenport's theorem in the theory of irregularities of point distribution, J. Math. Sci. (N. Y.) 115 (2003), no. 1, 2076-2084. MR.1805869 (2003d:11115)

6. J.G. van der Corput, Verteilungsfunktionen I, Nederl. Akad. Wetensch. Amsterdam Proc. 38 (1935), 813-821.

7. H. Davenport, On irregularities of distribution, Mathematika 3 (1956), 131-135. MR0082531 $(18: 566 \mathrm{a})$

8. J.H. Halton, S.C. Zaremba, The extreme and $L^{2}$ discrepancies of some plane sets, Monatsh. Math. 73 (1969), 316-328. MR0252329 (40:5550)

9. J. Matoušek, Geometric Discrepancy. An Illustrated Guide, Algorithms and Combinatorics, vol. 18, Springer-Verlag, Berlin, 1999. MR1697825 (2001a:11135)

10. P.D. Proĭnov, Symmetrization of the van der Corput generalized sequences, Proc. Japan Acad. Ser. A Math. Sci. 64 (1988), no. 5, 159-162. MR965955 (89i:11091)

11. K.F. Roth, On irregularities of distribution, Mathematika 1 (1954), 73-79. MR0066435 $(16: 575 \mathrm{c})$

12. K.F. Roth, On irregularities of distribution. III, Acta Arith. 35 (1979), 373-384. MR553291 (81a:10065)

13. K.F. Roth, On irregularities of distribution. IV, Acta Arith. 37 (1980), 67-75. MR598865 (82f:10063)

14. W.M. Schmidt, Irregularities of distribution. VII, Acta Arith. 21 (1972), 45-50. MR0319933 $(47: 8474)$

15. M.M. Skriganov, Harmonic analysis on totally disconnected groups and irregularities of point distributions, J. Reine Angew. Math. 600 (2006), 25-49. MR2283797(2007k:11122)

Department of Mathematics, University of South Carolina, Columbia, South CaroLINA 29208

Current address: School of Mathematics, Institute for Advanced Study, Princeton, New Jersey 08540

E-mail address: bilyk@math.ias.edu 